\title{
Scolia
}

Revue de linguistique

30 | 2016

Des connecteurs argumentatifs aux opérateurs discursifs

\section{Le savoir partagé comme source d'énonciation : les marqueurs je sais, tu sais, vous savez}

\section{María Luisa Donaire}

\section{(2) OpenEdition}

\section{Journals}

Édition électronique

URL : http://journals.openedition.org/scolia/484

DOI : $10.4000 /$ scolia.484

ISSN : 2677-4224

Éditeur

Presses universitaires de Strasbourg

Édition imprimée

Date de publication : 19 juin 2016

Pagination : 67-88

ISBN : 978-2-86820-944-3

ISSN : 1253-9708

Référence électronique

María Luisa Donaire, "Le savoir partagé comme source d'énonciation : les marqueurs je sais, tu sais, vous savez », Scolia [En ligne], 30 | 2016, mis en ligne le 09 avril 2019, consulté le 24 mai 2019. URL : http://journals.openedition.org/scolia/484 ; DOI : 10.4000/scolia.484 
SCOLIA 30/2016, p. 67-88.

\title{
Le savoir partagé comme source d'énonciation: les marqueurs je sais, tu sais, vous savez
}

\author{
María Luisa DoNAIRE \\ Université d'Oviedo, \\ groupe OPÉRAS \\ donaire@uniovi.es
}

\section{Introduction}

Cet article a pour objet l'analyse sémantique de deux marqueurs discursifs formés sur le verbe savoir: d'un côté tu sais/vous savez, reconnu par les linguistes et parfois mentionné par les dictionnaires et les grammaires ${ }^{1}$, mais aussi de l'autre je sais, qui n'apparait dans aucune liste des marqueurs formés sur savoir ${ }^{2}$, mais que l'observation d'un grand nombre d'occurrences permet de classer parmi les marqueurs discursifs d'attitude.

Dans des contextes comme (1), on observe un emploi de je sais qui s'écarte de la signification de savoir en termes de connaissance et qui rapproche cette forme de tu sais / vous savez en tant que marqueurs du discours :

1 C'est le cas dans Riegel et al. (1994: 36), qui inclut vous savez parmi les «termes qui ont pour fonction d'attirer ou de maintenir l'attention d'autrui $[. .$.$] et qui jouent le$ rôle de ponctuation du discours oral».

2 À ma connaissance, il n’y a que Anscombre (1981) qui ait considéré je sais comme un marqueur: il l'inclut parmi les marqueurs de dérivation illocutoire, à côté de je crois, je pense, j'espère, et d'autres verbes à la première personne, mais il ne fait que le mentionner. Dostie (2004) ne l'inclut pas dans la liste des marqueurs formés sur savoir. 
1) - Tu crois en Dieu? demanda Shirley à Joséphine.

- Ben, oui..., répondit Joséphine, étonnée par la question de Shirley. Je Lui parle, le soir. Je vais sur mon balcon, je regarde les étoiles et je Lui parle. Ça m'aide beaucoup...

- Poor you!

- Je sais. Quand je dis ça, les gens me prennent pour une demeurée. Alors je n'en parle pas. (Pancol, Les yeux jaunes des crocodiles, 2006)

Je sais admet difficilement ici la paraphrase par «j’ai la connaissance de», «j'ai appris que» ou «j'ai l'expérience que» et n'admet pas l'insertion d'un pronom (je le sais) qui reprenne l'objet du possible "savoir», qui serait en tout cas ici implicite.

Je postulerai donc que la base savoir à l'indicatif présent fournit à la langue non seulement les marqueurs de deuxième et quatrième personne tu sais et vous savez, mais aussi la forme de première personne je sais. Le verbe savoir se trouve justement parmi ceux qu'Andersen (1996) appelle «verbes parenthétiques» et Willems \& Blanche-Benveniste $(2010)^{3}$ des verbes «faibles » ou "à rection faible», qui sont à l'origine de ce type de formations.

Seront donc considérés ici je sais, tu sais, vous savez en tant que marqueurs discursifs et il s'agira de déterminer quelle est la spécificité de ces marqueurs, formés sur le verbe savoir, qui permet de les distinguer d'autres marqueurs similaires, formés sur d'autres verbes recteurs faibles.

Les formes tu sais / vous savez ont déjà suscité l'intérêt des linguistes, notamment de Davoine (1981) et Dostie \& De Sève (1999), et donc l'objectif de cet article sera de revisiter ces marqueurs et de décrire je sais en appliquant une optique théorique et méthodologique qui permette d'atteindre le niveau le plus profond de la signification de ces marqueurs ${ }^{4}$.

3 C'est le cas aussi dans Blanche-Benveniste \& Willems (2007).

4 Par ailleurs, Vincent (1993: 80-82) inclut tu sais/ vous savez parmi les "ponctuants du discours", des ponctuants "d'hésitation", intervenant dans la structuration du discours; l'auteure leur attribue d'ailleurs une fonction interactive qui consisterait à "maintenir le contact ou s'assurer de l'écoute de l'allocutaire». Mais cette description constitue la seule mention de ces marqueurs. Pour sa part, Andersen (2007: 27) considère ces unités comme faisant partie d'un groupe de «marqueurs discursifs propositionnels", et leur attribue trois fonctions: une "fonction de 
Il s'agira de vérifier l'hypothèse suivante: je sais, tu sais, vous savez sont des marqueurs discursifs, dans la mesure où ils marquent une attitude du locuteur (désormais L) à l'égard de son allocutaire, attitude qui ne consiste pas simplement à montrer ou à partager une connaissance, ni à s'assurer de l'écoute, mais à établir un certain rapport énonciatif qui marque la participation active de l'allocutaire dans la construction du sens: cette attitude se manifeste moyennant une stratégie discursive qui consiste dans un arrangement particulier de points de vue 5 .

L'analyse consistera à décrire les propriétés linguistiques, tant formelles que sémantiques, de ces unités, afin de montrer ce qu'elles ont en commun et quelle est la signification spécifique à chacune d'entre elles. En particulier, dans le cas de je sais, l'analyse essaiera de montrer qu'il s'agit effectivement d'un marqueur discursif. Pour cela, je ferai appel à la version radicale de la théorie de la polyphonie ${ }^{6}$, qui postule essentiellement que la structure profonde de tout énoncé se décompose en une série d'entités abstraites, des points de vue, notés $p d v$, comportant une source et un objet construit (ou contenu). Ces $p d v$ sont interprétés, en surface, comme des voix identifiant plusieurs auteurs de discours.

Sera d'abord décrit le comportement distributionnel des unités choisies, le niveau superficiel, pour atteindre ensuite la structure sémantique profonde qui explique le fonctionnement sémanticopragmatique de ces marqueurs, en termes de stratégie.

structuration du discours aux niveaux syntaxique, thématique et discursif», une "fonction phatique qui peut être la recherche d'approbation discursive, la marque de politesse ou de camaraderie» et «une fonction d'instrument de prise de tour». Par manque d'espace, je ne commenterai ni critiquerai ici en détail ces diverses études. En général, ou bien il n'y a pas de critères qui justifient la description, ou bien ces critères sont insuffisants ou ils s'appuient sur une composante informative du sens.

5 Ils s'apparentent en ceci à d'autres marqueurs d'attitude comme je dirais, je ne saurais dire, comment dirais-je. Pour une analyse polyphonique de ces marqueurs, se reporter à Donaire (à paraître).

6 Pour une description détaillée de la théorie, se reporter notamment à Anscombre (2013). 


\section{Tu sais/vous savez}

La différence entre tu sais et vous savez n'est que formelle, elle ne correspond pas à une différence de sens: on emploie l'une ou l'autre selon que le locuteur s'adresse à un ou à plusieurs interlocuteurs, ou que la relation entre le locuteur et l'interlocuteur soit de tutoiement ou de vouvoiement ${ }^{7}$. C'est pourquoi ces deux unités seront décrites ensemble et désignées de façon indistincte par la forme $t u$ sais.

On partira de l'hypothèse, en accord avec Davoine (1981), que le marqueur tu sais recouvre deux entités sémantiques, désignées ici comme $t u$ sais $_{1}$ et $t u$ sais $_{2}{ }^{8}$, qui partagent le contexte (2), et on essayera de vérifier ensuite l'existence d'une troisième entité à valeur purement interactive ${ }^{9}$ :

2) Heureusement Marie a pris ce job au centre commercial. Elle est serveuse au bar, tu sais $\boldsymbol{s}_{1}$, celui où on allait après le ciné. Sans ça, je ne sais pas comment on aurait fait. Mais là, tu vois, on tient le bon bout. On gagne pas lourd ni l'un ni l'autre mais ça va on s'en sort. Et puis les gamines sont super. Elles ne réclament jamais rien. Elles ne se plaignent jamais. Mais tu sais şa me fait mal au bide qu'on ait régressé comme ça. Je veux dire, normalement, ça se passe dans l'autre sens, non? (Adam, Les lisières, 2012)

Mis à part les propriétés communes qui font de $t u$ sais $_{1}$ et de $t u$ sais $_{2}$ un marqueur, chaque entité sémantique se distingue par des propriétés spécifiques qu'on analysera par la suite ${ }^{10}$. De façon très synthétique, tu sais $s_{1}$ adapte le contenu de l'énoncé au savoir de l'allocutaire, tandis que $t u$ sais $_{2}$ introduit un commentaire de L s'opposant au discours qui précède le marqueur.

7 D'après Dostie \& De Sève (1999), t'sais ne commuterait pas avec vous savez (ni même avec tu sais) dans certains de ses emplois, concrètement lorsqu'il a la valeur de vérificateur, d'évaluatif ou de pisteur.

8 Le recours à des indices numériques pour désigner les diverses entités sémantiques prétend éviter les étiquettes, qui ne décriraient que partiellement la signification des unités.

9 Pour Vincent (1993) "ponctuant»; dans Dostie \& De Sève (1999) «pisteur»; «de balisage» pour Dostie (2004).

10 L'analyse de l'écrit et de l'oral (je n'aborderai pas ce deuxième aspect ici) aboutit aux mêmes résultats. 


\subsection{Tu sais}

3) - Mais tu ne le reconnais pas, Eliott? C'est Gabriel Askolein, tu sais $_{1}$, l'écrivain de science-fiction. (Werber, Le mystère des dieux, 2007)

\subsubsection{Propriétés distributionnelles}

Ce marqueur répond à une structure formelle de type $X$, tu sais, $Y, X$ étant nécessairement constitué par un syntagme et $Y$ pouvant être un syntagme, comme dans (2) et (3), ou un énoncé comme dans (4):

4) Elle est intolérable, l'image de cet enfant enfermé dans un sac, attaché, si ça se trouve, avec une espèce de fil rouge, vous savez, comme celui qu'il y a autour des portions de crème de gruyère.

(Desproges, Chroniques de la haine ordinaire, 1997)

Dans (3), le syntagme à la place d'Y est une expression définie de forme le+nom, ce dernier suivi d'une expansion; dans (2) et (4) l'expression est de forme pronom démonstratif+nom+expansion relative (celui où/celui qu'il y a) - dans (4) l'énoncé est d'ailleurs introduit par le comparatif comme, ce qui est aussi fréquent - ; dans (5) il s'agit de déterminant démonstratif +nom+expansion relative:

5) - Je crois qu'on va aller faire un petit tour chez les Amishs. Tu sais, ces gens merveilleux qui vivent sans téléphone ni électricité, comme au siècle dernier? (Jubert, Le virus de décembre, 1999)

Ce marqueur se caractérise par la position en incise ${ }^{11}$ exclusivement médiane et, face à d'autres marqueurs, il apparaît toujours en dialogue.

À l'écrit, il peut être suivi d'une marque d'intonation interrogative:

6) - Ce rôle de jouet m'insupporte. Ça me fait penser au Prisonnier, tu sais? cette série où des gens sont coincés quelque part dans un hôtel de vacances et où le héros répète "je ne suis pas un numéro». (Werber, Nous les dieux. L'île des sortilèges, 2004)

\subsubsection{Propriétés sémantico-pragmatiques}

La structure sémantique de ce marqueur est toujours de forme $p$ tu sais s $_{1} q$ se présentant comme une paraphrase de $p$, de telle façon

11 Pour Riegel et al. (1994: 461) il s'agit de «propositions incidentes». 
que $p$ tu sais s $_{1} q$ admet la transformation en une phrase attributive de forme $p$ est $q$ :

2') le bar est celui où on allait après le ciné.

3') Gabriel Askolein est lécrivain de science-fiction.

4') le fil rouge est comme celui qu'il y a autour des portions de crème de gruyère.

5') les Amishs sont ces gens merveilleux qui vivent sans téléphone ni électricité.

6') le Prisonnier est cette série où des gens sont coincés quelque part dans un hôtel de vacances.

Le fait que tu sais ${ }_{1}$ commute avec c'est-à-dire confirme également la relation paraphrastique entre $p$ et $q$ : par exemple, (3) Gabriel Askolein, c'est-à-dire l'écrivain de science-fiction. Par ailleurs, cette paraphrase $q$ apparaît comme un commentaire de L à propos de $p$ et à l'adresse du destinataire, celui-ci étant présenté comme directement et explicitement concerné par l'énonciation, tout comme dans un énoncé interrogatif. L'énonciation de $q$ est justement motivée par un $p d v$ attribué à l'allocutaire.

L'article le ou d'autres expressions définies dans $q$, présentent $q$ comme connu par le destinataire ${ }^{12}$, et donc comme un «savoir partagé ${ }^{13}$ par L et l'allocutaire. Par contre, $p$ est présenté comme un $p d v$ non partagé au moment où il est énoncé, ce qui motive justement l'énonciation de $q$.

Tu sais ${ }_{1}$ marque donc une réaction de $\mathrm{L}$ à l'égard de sa propre énonciation $(p)$, construisant par là une représentation de l'allocutaire comme ne partageant pas son espace discursif et cherchant à l'intégrer à cet espace en énonçant ensuite $q$. Un argument en faveur de cette représentation de l'allocutaire est la possibilité de combiner tu sais avec mais si, comme dans (7), si exigeant la présence d'un argument négatif préalable:

12 Cette propriété mène Davoine (1981: 111) à considérer que tu sais «d'identification " peut commuter avec tu sais bien. Cependant, on ne peut considérer les deux marqueurs comme synonymes. Par manque d'espace, je ne traiterai pas ici cette différence, qui réside fondamentalement dans la présence de bien.

13 Cela a été remarqué par Dostie \& De Sève (1999: 16) : «T'saisI.1 mise sur l'existence d'un savoir partagé, antérieur à l'échange en cours». Mais l'analyse entreprise ici montre que la dynamique mise en jeu par $t u s^{s a i s_{2}}$ est bien plus compliquée. 
7) Je lui ai décrit mon butin par le menu, pas de petite robe, mais une paire d'escarpins à tomber, des barrettes adorables et des dessous trop jolis, mais si, tu sais, un soutif comme ceux de chez Eres, avec les bonnets comme ci et les bretelles comme ça, des petites culottes [...]. (Gavalda, La vie en mieux, 2014)

Si on élimine le marqueur, la représentation de la réaction à l'égard de $p$ disparaît, $q$ se présentant comme une simple paraphrase ou reformulation de $p$ :

3a) Mais tu ne le reconnais pas, Eliott? C'est Gabriel Askolein, l'écrivain de science-fiction.

La stratégie consiste donc à établir la relation entre au moins trois $p d v: p d v_{1}$, dont la source est L et l'objet construit $\{p\} ; p d v_{2}$ qui présente $p d v_{1}$ comme non partagé par L et l'allocutaire et dont la source est l'allocutaire; $p d v_{3}$ dont la source est L et l'objet construit $\{q\}$ présenté comme partagé par L et l'allocutaire.

\subsection{Tu sais}

8) Il me proposa à boire. Je lui demandai du coca. Il eut un air surpris.

- Désolé. Je suis d'une ancienne génération, vous savez. Je ne bois que de l'alcool. (Humbert, L'origine de la violence, 2010)

\subsubsection{Propriétés distributionnelles}

Ce marqueur répond à une structure formelle de type $X$, tu sais $s_{2}$, comme dans (8), ou tu sais $2, X$, comme dans (9), X étant nécessairement constitué par un énoncé:

9) - Je ne peux rien dire. Bon, assez bavardé, je vous raccompagne, il est tard.

- Tu sais, Can, je suis une grande fille et tu n'es pas obligé de m'escorter tous les soirs jusque chez moi. (Lévy, L'étrange voyage de Monsieur Daldry, 2011)

Les énoncés auxquels $t u s{ } i_{2}$ peut être associé présentent quelques particularités: des marques de la première personne, comme dans (2), (8) et (9); ou bien les pronoms on, nous incluant je mais pas nécessairement $t u$, comme dans (10) et (11):

10) Vous faites quelque chose le soir du 31 ? s'enquit Jed. 
- Non, rien de particulier; je relis Tocqueville en ce moment. Vous savez, à la campagne, on se couche tôt, surtout en hiver. (Houellebecq, La carte et le territoire, 2010)

11) L'albinos ne semble pas satisfait de ma réponse.

- Tu sais, Eliott, nous avons un problème avec le moteur d'intelligence artificielle qui va contrôler les déplacements des personnages en Olympie. (Werber, Le mystère des dieux, 2007)

De même, le sujet de la phrase peut être tu mais, dans ce cas, je apparaît également sous l'expression «tu n'étais pas la seule» car on comprend «moi aussi» :

12) «Nous n'avons jamais rien su de ce qu'il était advenu de ta mère. Tu sais, tu n'étais pas la seule à être en danger. " (Lévy, L'étrange voyage de Monsieur Daldry, 2011)

Lorsque le sujet est de troisième personne et la phrase est au présent de l'indicatif, elle se présente comme une phrase générique ${ }^{14}$ :

13) Au fond, Harry, comment devient-on écrivain?

- En ne renonçant jamais. Vous savez, Marcus, la liberté, l'aspiration à la liberté est une guerre en soi. (Dicker, La vérité sur l'affaire Harry Quebert, 2012)

14) - Tu sais, il y a des gens à qui la vie, telle qu'elle est, suffit. J'ai mis longtemps à le comprendre. Quel mal y a-t-il à mener une vie normale? (Adam, Les lisières, 2012)

Dans (15), le verbe est au passé et à la troisième personne, mais le démonstratif, déictique, marque la référence à la première personne:

15) Tu sais, dans cette ville aux cantonales le Front national a fait $30 \%$. À ton avis qu'est-ce que ça leur envoie comme message, aux gamins? On veut pas de vous. (Adam, Les lisières, 2012)

Il est également fréquent de trouver des modalisateurs dans l'énoncé $X$ auquel s'associe $t u$ sais 2 , que ce soit des verbes modaux

14 Setterkorn (1977: 203) établit un certain rapport entre quelques marqueurs, dont vous savez, et les "propositions généralisantes": "Les particules et tournures à fonction r.a.d. [recherche d'approbation discursive] présentent des structures argumentatives; ce sont de courtes formes d'argumentation; du fait qu'elles élèvent une exigence de validité (plus ou moins grand $[s i c]$ ) pour la proposition à laquelle elles se rapportent, elles peuvent être intégrées dans de plus amples contextes argumentatifs en tant que propositions justificatives. Dans cet ordre d'idées, ce sont des correspondants fonctionnels aux propositions généralisantes.» 
comme je crois, dans (16), ou des expressions comme mieux vaut et peut-être, dans (17), ou la présence du futur, comme dans (18), accompagné aussi de peut-être, dans ce cas:

16) - Franchement, je reste étonné qu'il en ait gardé autant.Pour être vraiment apprécié des gens, tu sais, je crois qu'il faudrait mourir tout le temps. (Giesbert, L'immortel, 2007)

17) Et me voilà bricolant dans son chagrin. Mieux vaut, lui dis-je très prudemment, peut-être, le divorce, tu sais, Jocelyne, enfin... deux divorcés apaisés te seront plus supportables qu'un couple acharné à se détruire... (Pennac, Chagrin d'école, 2007)

18) - Je suis désolé pour ton père, me dit-il. Tu sais, avec le temps, les choses finiront peut-être par s'arranger. (Lévy, Le voleur d'ombres, 2010)

Ce marqueur se caractérise par sa position en incise mais surtout, contrairement à tu sais $s_{1}$, par sa mobilité, car il peut occuper la position initiale, qui prédomine, comme dans (9) à (15) et (18), mais aussi finale, comme dans (8) et (17), et même parfois médiane, comme dans (16). Tout comme tu sais $s_{1}$, tu sais $s_{2}$ apparaît toujours en contexte dialogique.

Par ailleurs, à l'écrit, il peut être suivi d'une marque d'intonation interrogative, cf. (19), ou exclamative, cf. (20):

19) Et vous n'aimez pas les gens comme nous, parce qu'on ne vous aide pas à justifier ce que vous avez fait, qu'on ne vous glorifie pas de l'avoir fait. Mais moi je vous pardonne, vous savez? (Dugain, Avenue des géants, 2012)

20) - Charles et Louis-Marie sont complètement désemparés. Ils ont besoin de toi, Jean-Hugues.

-Et que veux-tu que je fasse? Je ne peux pas la ressusciter, tu sais!

- Je t'interdis de blasphémer! (Aubert, Funérarium, 2002)

Il se produit parfois l'ellipse de $X$ lorsque celui-ci devrait suivre le marqueur:

21) - Vous n'êtes pas en vacances?

- Je n'ai pas vraiment d'emploi, alors vous savez, les vacances...

(Lévy, L'étrange voyage de Monsieur Daldry, 2011) 


\subsubsection{Propriétés sémantico-pragmatiques}

La structure sémantiquede ce marqueur est toujours de forme $p$ tu sais $s_{2} q$, où $p$ et $q$ représentent deux discours que le marqueur met en relation. La position du marqueur n'est qu'un fait de surface, l'agencement sémantique étant toujours de forme $p$ tu sais $q$, le marqueur établissant un lien sémantique entre $p$ et $q$ qui, contrairement à $t u$ sais $s_{1}$, n'est pas présenté comme donné d'avance.

Dans (9), par exemple, la structure de surface est de forme tu sais, $X$ ( $X=j$ je suis une grande fille et tu n'es pas obligé de m'escorter tous les soirs jusque chez moi), mais la structure sémantique est de forme $p$ tu sais $s_{2} q$ ( $p=$ je vous raccompagne; $q=\ldots$ tu n'es pas obligé de m'escorter...), car tu sais $_{2} q$ ne peut pas être compris indépendamment de $p$.

Quant au rapport entre $p$ et $q, q$ se présente comme un commentaire de $\mathrm{L}$ à propos de $p$ (un $p d v$ ayant une source différente de L). De là, les marques fréquentes de la première personne, sous la forme pronominale, ou moyennant l'emploi de modalisateurs ou de déictiques.

Pour déterminer quel type de commentaire introduit L, il faut signaler certaines propriétés des contextes de $t u$ sais $_{2}$. On observe que ce marqueur apparaît souvent en contexte négatif explicite, c'est-à-dire présentant des morphèmes négatifs dans $p$ (ex. 2, 10,11) ou dans $q$ (ex. 9,11 ), et même dans $p$ et $q$, comme dans (12). Dans (8), la négation apparaît dans l'enchaînement qui explique $q$.

Cette négation peut par ailleurs se doubler d'une opposition, marquée par mais précédant immédiatement $t u s_{a i s_{2}}$, comme c'est le cas dans (2). Mais elle peut aussi rester implicite, ou bien parce qu'elle se manifeste sous forme d'opposition (mais), comme dans (22) - où il y a aussi le verbe éviter, à sens négatif -, ou parce qu'elle fait partie de l'interprétation de $p$ : dans (18), par exemple, les choses finiront peut-être par s'arranger fait entendre les choses ne sont pas arrangées maintenant:

22) - Dans quelle chambre se trouve Nola? Je voudrais aller lui dire bonjour.

- C'est très aimable à vous, mais vous savez, il est préférable qu'elle évite les visites pour le moment. Il ne faut pas qu'elle se fatigue, vous comprenez. (Dicker, La vérité sur l'affaire Harry Quebert, 2012) 
Dans (22), q équivaut à une négation: n'allez pas lui dire bonjour, ici par la présence de mais. Mais c'est aussi le cas partout: avec tu sais $_{2} \mathrm{~L}$ plaide pour une conclusion contraire à celle qu'on peut tirer de $p$ : cf. (8) «du coca»/ «que de l'alcool»; (10) «faire quelque chose le soir» / «se coucher tôt».

On peut donc conclure que la source du $p d v$ représenté par $q$ est nécessairement distincte de celle du $p d v$ représenté par $p$. La source de $p$ est un ex-locuteur identifié au destinataire (interlocuteur dans le dialogue). Quant à $q$, lorsqu'il est constitué par une phrase générique, la source est identifiée à la communauté linguistique, un ON-locuteur auquel $\mathrm{L}$ dit appartenir ainsi que son allocutaire, tandis que, lorsqu'il y a des marques de première personne, des déictiques, des modalisateurs, on serait tentés de l'attribuer à L. Mais, l'examen attentif des contextes fait dériver plutôt la source vers la communauté linguistique. On va l'expliquer en reprenant le $t u$ sais $_{2}$ de l'exemple (2):

2) Elles ne réclament jamais rien. Elles ne se plaignent jamais. Mais tu sais ça me fait mal au bide qu'on ait régressé comme ça. Je veux dire, normalement, ça se passe dans l'autre sens, non?

On a en effet des marques de première personne me, on. Mais L explique par la suite pourquoi cela lui fait mal (je veux dire) en faisant recours à une norme (normalement), à ce qui est accepté par la communauté linguistique ${ }^{15}$, et il demande l'accord de l'interlocuteur (non?), le présentant par là comme appartenant à cette communauté linguistique.

Et tous ces contextes peuvent être expliqués de la même façon: (8) l'ancienne génération boit de l'alcool; (9) une grande fille peut marcher toute seule le soir; etc. La source n'est donc pas L mais un ON-locuteur. Le fait qu'il soit possible de faire l'ellipse de $q$ en est un argument, la suite pouvant être facilement reconstruite puisqu'il s'agit d'un savoir partagé par la communauté linguistique. Quant aux contextes où il y a des modalisateurs, ce sont des marques de prise en charge d'un $p d v$ dont la source est la communauté linguistique à laquelle appartiennent L et l'allocutaire.

L'allocutaire est donc toujours concerné, tout comme avec tu sais $s_{1}$, la différence entre les deux marqueurs résiderait dans le fait que $t u$ sais

15 Un stéréotype, dans le sens défini par Anscombre (2001). 
présente l'allocutaire comme ne partageant pas l'espace discursif de L, tandis que tu sais $s_{2}$ le présente comme partageant cet espace commun ${ }^{16}$.

La stratégie propre à $t u s a i_{2}$ consiste donc à établir la relation entre au moins quatre $p d v: p d v_{1}$, dont la source est un ex-locuteur et l'objet construit $\{p\} ; p d v_{2}$ dont la source est L et qui se présente comme opposé à $p d v_{1}\{$ non $p\} ; p d v_{3}$ dont la source est un ON-locuteur et l'objet construit $\{q\}$; $p d v_{4}$ dont la source est L et consiste dans la prise en charge de $p d v_{3}$.

\subsubsection{Y aurait-il un tu $\mathrm{sais}_{3}$ ?}

Pour Vincent (1993), Dostie \& De Sève (1999), et Dostie (2004), parmi d'autres ${ }^{17}$, il y aurait un emploi de $t u$ sais distinct de ceux qui sont décrits ci-dessus, et qui ne servirait qu'à "ponctuer», ou comme «pisteur», ou à «baliser» le discours. Ce serait une valeur caractérisant essentiellement l'oral et dont les propriétés seraient: possibilité d'être répété ou cumulé dans une même énonciation et position en finale de syntagme ou d'énoncé. Pour Dostie \& De Sève (1999: 30), il s'agirait d'un «marqueur discursif discontinu», ce qui expliquerait sa récursivité.

Mais l'analyse d'un nombre important d'occurrences dans des corpus oraux ${ }^{18}$ ne nous a pas permis d'établir des propriétés spécifiques distinctes de celles que présentent $t u$ sais $_{1}$ et $t u$ sais $_{2}$. Par ailleurs, dans les contextes proposés par les auteurs cités, les occurrences de tu sais peuvent être facilement analysées comme des cas de $t u$ sais $_{1}$ ou de $t u$ sais $_{2}$. C'est bien le cas, par exemple, du contexte ci-dessous:

23) Moi, quand j'étais jeune, mon amie s'est faite frapper par un train, oui, pi elle est morte à part ça. Ah mon Dieu, ça m'a impressionné! J'ai ouvert la radio à grandeur pour savoir t'sais

16 Andersen (2007: 19-20) décrit ainsi le sens de tu sais/vous savez: «Prototypiquement, ce MDP [marqueur discursif propositionnel] indique que le but du locuteur est de faire coopérer l'interlocuteur ou de lui faire accepter le contenu propositionnel de son énoncé comme un savoir commun.»

17 Par exemple, pour Traverso (1996: 135) t'sais fait partie «des marqueurs de structuration de la conversation ».

18 Environ 400 occurrences extraites des bases de données CLAPI, Corpus de la parole, ELICO et ESLO. 
[1] ${ }^{19}$, voir si elle était réellement morte t'sais [2]. Mais je crois que je vais dire comme ma mère: avec les années ça s'oublie t'sais [3], mais sur le coup t'sais [4], ça nous laisse un peu... t'sais? [5], hein? (Dostie \& De Sève, 1999: 23).

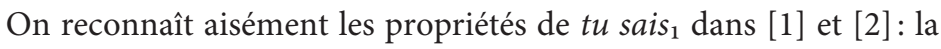
présence d'expressions définies; $p$ est un syntagme (pour savoir) et $q$ un énoncé (voir si elle était réellement morte); le rapport entre $p$ et $q$ est une relation de paraphrase, ce qui rend possible la transformation en phrase attributive et/ou la commutation par c'est-à-dire: "savoir est voir si elle est réellement morte»; "J'ai ouvert la radio à grandeur pour savoir c'est-à-dire pour voir si elle était réellement morte». Par ailleurs, L est source de $p$ et $q$. Quant à [3], [4] et [5], ils répondent aux propriétés de tu sais $s_{2}$ p et $q$ sont des énoncés ( $p=$ avec les années ça s'oublie; $q=$ sur le coup ça nous laisse un peu...) ; présence de marques de première personne (je crois, je vais dire, ma mère, nous); présence d'une phrase générique (le temps fait l'oubli); présence de mais; ellipse de $q$ dans [5]; $q$ introduit un commentaire à propos de $p$; la source de $q$ est la communauté linguistique à laquelle appartiennent L et son allocutaire, ce qui est marqué par la présence de hein?

Les différences qu'on aperçoit ne me semblent pas le fait du marqueur, mais plutôt de l'expression orale, beaucoup plus elliptique, laissant beaucoup plus de place à l'implicite parce que les vides peuvent être aisément remplis par la situation, et plus spontanée, ce qui peut affecter la mobilité des marqueurs et leur récursivité.

\section{Je sais $^{20}$}

1) - Tu crois en Dieu? demanda Shirley à Joséphine.

- Ben, oui..., répondit Joséphine, étonnée par la question de Shirley. Je Lui parle, le soir. Je vais sur mon balcon, je regarde les étoiles et je Lui parle. Ça m'aide beaucoup...

- Poor you!

19 La numérotation a été ajoutée ici pour identifier les formes dans l'analyse qui va suivre.

20 Même si, dans le français actuel, la forme je sais ne correspond qu'à une seule entité sémantique, le chiffre dans je sais $s_{1}$ signale que la description correspond à cette valeur actuelle et n'exclut pas le développement d'autres valeurs dans le futur, qui porteraient alors un chiffre différent. 
- Je sais. Quand je dis ça, les gens me prennent pour une demeurée. Alors je n'en parle pas. (Pancol, Les yeux jaunes des crocodiles, 2006)

\subsection{Propriétés distributionnelles}

Ce marqueur répond à une structure formelle de type $(X)$, je sais $s_{1}$, $(Y)$, c'est-à-dire que l'on peut trouver la structure $X$, je sais $s_{1}$, comme dans (24), je sais,$Y$, comme dans (25), ou bien je sais, comme dans (1):

24) - C'est ma collègue qui l'ouvrira mais, vu l'impact, le carreau a traversé le larynx jusqu'à l'œesophage. Je pense qu'il est mort d'étouffement avant que l'hémorragie n'ait fait son œuvre. On le rhabille?

- On ne peut pas, docteur. Faut que les techniciens passent.

- Quand même, dit Merlan avec une grimace.

- Oui, docteur, je sais. (F. Vargas, L'armée furieuse, 2011)

25) -Je vais commencer le théâtre, tu sais? poursuivit-elle. J'ai été repérée par Cheryl Crawford, la directrice du théâtre de Maplewood. C'est pour ça, la Débutante, la Glamour Girl... c'est comme quand on s'est embrassés tout à l'heure, je me laisse porter. C'est ridicule, je sais, mais c'est mieux que de continuer à étudier des sottises qui ne me serviront jamais à rien. (F. Beigbeder, Oona \& Salinger, 2014)

Dans ces structures formelles, tant je sais $s_{1}$ que $X$ et $Y$ représentent des énoncés, c'est-à-dire que je sais ${ }_{1}$ constitue à lui tout seul un énoncé dont ni $X$ ni $Y$ ne font partie.

Bien que je sais $s_{1}$ peut constituer à lui tout seul une énonciation, comme dans (1), ce marqueur admet également la position en incise, initiale le plus fréquemment, comme dans (26), mais aussi finale, comme dans (24), ou médiane, comme c'est le cas dans (25):

26) En me réveillant, j'aperçus Neumann qui examinait la toile dans la lumière du jour. La honte me tomba dessus et je fis semblant de dormir encore. Mais Neumann ne partait pas.

Il restait en face du tableau, il l'examinait, il létudiait.

- Je sais, c'est ridicule! lui lançai-je de loin. (Schmitt, La part de l'autre, 2001) 
Ce marqueur s'emploie toujours en dialogue, même si ce dialogue est virtuel, comme c'est le cas dans (27), où L s'adresse à un interlocuteur qui n'est pas présent au moment de l'énonciation:

27) J'aurais bien aimé être comme elle et en même temps non. J'ai une part de calme que j'aime bien. Je sais, monsieur le Psy, je sais. Calme. Son côté à lui. Ce qui m’attache à lui. Ce qui me rapproche de lui. (Délacourt, On ne voyait que le bonheur, 2014)

Souvent, je sais $s_{1}$ est suivi de l'interpellation à l'interlocuteur, en apposition, comme dans (24), docteur, et (27), monsieur le Psy; dans (28) c'est le nom propre:

28) - Mais te rappelles-tu ce jour, sur la plage, après le spectacle, lorsque tu m'as dit de ne pas m'en faire puisque tu étais là? Comment veilleras-tu sur moi si tu te tues?

- Je sais, Harry. Pardon, je vous demande pardon. (Dicker, La vérité sur l'affaire Harry Quebert, 2012)

Je sais $s_{1}$ présente la particularité de pouvoir se doubler, tel que dans (29), propriété qu'il ne partage pas avec tu sais:

29) Ça m'a tellement gavée que j'ai fini par lui refourguer un énorme bobard (je sais, je sais, je n'ai aucun mérite, c'est mon métier) pour ne pas prendre le risque de l'envoyer chier grave. (Gavalda, La vie en mieux, 2014)

Ce marqueur n'admet pas des insertions, notamment un pronom ( ${ }^{*}$ je le sais); il ne peut pas être précédé de et ( ${ }^{*}$ et je sais) et il n'apparaît qu'en phrase assertive ${ }^{21}$. À l'écrit, il peut être suivi d'une marque d'intonation exclamative:

30) - Pourquoi tu me dis tout ça, maintenant?

- Pour t'éclairer sur qui tu étais vraiment. Ce jeune homme sage que j'adorais.

- Ah... oui, moi aussi, je t'adorais...

- Je sais! Bon, assez parlé de toi... passons aux choses sérieuses!

(Foenkinos, Je vais mieux, 2014)

Je sais ${ }_{1}$ est souvent précédé de oui, (24) et (31), et suivi de mais, (25) et (29), et même encadré par les deux morphèmes, comme dans (32):

21 Il y a lieu de se demander si je sais bien et je (ne) sais pas, qui présentent un comportement formel similaire à celui de je sais $s_{1}$, peuvent être considérés comme marqueurs discursifs, mais ce sera peut-être l'objet d'un autre article. 
31) Sortit un long cigare qu'il renifla et fit rouler entre ses doigts avant de l'allumer et ajouta:

- Oui, je sais, on ne fume plus dans les restaurants. Sauf moi. J'emmerde les lois. (Pancol, Les écureuils de Central Park sont tristes le lundi, 2010)

32) Il faut peut-être que tu te trouves un autre ami...

- C'est lui que je veux, maman!

- Oui, je sais, mais vous n'êtes pas du tout sur la même longueur d'onde. Il faut que tu t'éloignes pour que tu lui redeviennes précieuse. (Pancol, Les yeux jaunes des crocodiles, 2006)

\subsection{Propriétés sémantico-pragmatiques}

La structure sémantique de ce marqueur est toujours de forme $p$ je sais $_{1} q$, où $p$ et $q$ représentent deux discours que le marqueur met en relation. La position du marqueur n'est qu'un fait de surface.

Dans cette structure, qui correspond à l'agencement sémantique, il ne s'agit pas de je sais $p$ ou je sais $q$, c'est-à-dire que $p$ et $q$ ne se présentent pas comme l'objet de savoir, mais je sais $s_{1}$ constitue une réaction à l'égard de $p$, réaction qui peut être explicitée par $q$. La différence entre (33a) et (34a) réside dans la difficulté pour (34a) d'enchaîner sur (34b):

33) a. Je sais, c'est ridicule.

b. Je sais, c'est ridicule. Je sais.

34) a. Je sais que c'est ridicule.

b. Je sais que c'est ridicule. ??Je sais.

Par ailleurs, la réponse affirmative à une question à propos d'un «savoir» ne peut pas être je sais ${ }_{1}$ tout court, ce marqueur étant toujours la réplique à une phrase assertive:

35) -Tu sais conduire?

-??Je sais.

36) - Tu sais conduire.

- Je sais.

Donc, je sais $s_{1}$ peut servir à confirmer une affirmation $p$ de l'interlocuteur mais ne peut pas être l'équivalent d'une réponse confirmant $p$. Cela s'explique parce que la réaction à un discours 
précédent $p$, marquée par je sais $s_{1}$, se présente dans un premier moment comme un accord avec $p$ (un $p d v_{1}$ pris en charge par L, dans (36) «je sais conduire»), ce qu'on aurait dans le cas de (35), mais je sais introduit en même temps un argument $q$ pour s'opposer à $p$ (un $p d v_{2}$ qui s'oppose à $p d v_{1}$, dans (36) «je n'assume pas les conclusions qu'on peut tirer de $p d v_{1}$ ", par exemple «conduire ta voiture»). Le comportement différent de je sais dans (37a) et (37b) montre cette particularité:

37) a. Je sais que tu vas avoir un bébé, quel bonheur!/ quel problème!

b. Je sais, tu vas avoir un bébé, ??quel bonheur!/ quel problème!

En effet, je sais pouvant être suivi d'une complétive et ayant donc le sens de savoir, admet tant des environnements positifs que négatifs, tandis que je sais $s_{1}$ marqueur semble réfractaire aux environnements positifs. Les occurrences du corpus, et les exemples proposés ici, confirment cette hypothèse: c'est ridicule, dans (25) et (26); quand même dans (24); pardon dans (28) ${ }^{22}$; je n'ai aucun mérite dans (29); assez parlé dans (30); on ne fume plus dans (31); vous n'êtes pas du tout dans (32). Cet environnement négatif n'est nullement justifié par le sens de savoir, ce qui fournit un argument pour le considérer comme un marqueur.

Dans ce sens, il est à signaler la présence fréquente de mais enchaînant sur je sais $s_{1}$, qui lui prête une certaine valeur concessive: dans (25), "C'est ridicule, je sais, mais c'est mieux que de continuer à étudier des sottises qui ne me serviront jamais à rien" admet la paraphrase concessive Même si c'est ridicule, c'est mieux que de continuer à étudier des sottises...

On pourrait objecter que ce n'est pas toujours le cas, qu'il y a des contextes où on ne perçoit pas ce négatif, où je sais $s_{1}$ semble favoriser plutôt l'interprétation comme une marque d'accord avec la source de $p$, interprétation renforcée par la présence fréquente de oui à gauche du marqueur. C'est ce qu'on pourrait dire aussi de (38) et (39) où, apparemment il n'y a pas de signes de désaccord:

38) Sans rien avoir demandé, elle se retrouva une coupe de Champagne dans une main, un blini nappé de caviar dans

22 Dans le cas de (27) le contexte est insuffisant pour vérifier que "Calme. Son côté à lui.», etc., confirme également l'hypothèse: ce lui est le père du personnage dont le calme lâche l'a marqué. 
l'autre. Elle tourna un instant sur elle-même, hébétée, avant qu'on ne lui saisisse le coude. Brad lui faisait face.

- Je sais, murmura-t-elle, t'as failli attendre. Le taxi, c'était galère! (Jonquet, Ad vitam aeternam, 2006)

39) Elle se pencha vers les petits corps martyrisés, en effleura un du bout des doigts.

- Il faut qu'on le trouve, Chib.

- Je sais. (Aubert, Funérarium, 2002)

En effet, je sais ${ }_{1}$ semble ici commuter avec d'accord, mais cette commutation ne suffit pas à décrire la signification du marqueur, il faut y ajouter un élément qui reste implicite. Dans (38), la suite t'as failli attendre fait interpréter je sais ${ }_{1}$ comme la confirmation qu'elle devrait s'excuser mais elle a l'alibi du taxi. Je sais $s_{1}$ s'appuie donc ici sur une norme, un stéréotype (dont la source est donc un $O N$-locuteur) de forme "quand on est en retard il faut s'excuser", une paraphrase de (38) pouvant être: "d'accord, je suis en retard et je devrais m'excuser, mais ne me demande pas de m'excuser car le taxi [...]».

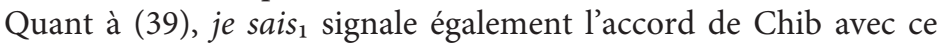
qu'elle dit, mais il y a quelque chose d'autre, il ne s'agit pas de je sais qu'il faut qu'on le trouve, la réplique aurait dû être alors je le sais, mais il s'agit de tu n'as pas besoin de me dire qu'il faut qu'on le trouve.

En effet, cela permet de préciser en quoi consiste 's'opposer à $p$ ': L ne s'oppose pas à ce qui est dit, mais au dire, à l'énonciation de $p$. Dans (40) et (41), cette interprétation est explicite:

40) - Eh bien... Merci, mon vieux, pour ces bonnes nouvelles et joyeux Noël!

Stanislas rit à l'autre bout du fil, puis reprit:

- Je sais, je ne devrais pas parler de cela ce soir, mais j'en ai marre d'entendre dire par tous ces imbéciles que la crise est derrière nous alors qu'elle vient juste de commencer. (Pancol, Les écureuils de Central Park sont tristes le lundi, 2010)

41) J'ai replié la voilure et rembobiné le fil. Puis j'ai demandé à Luc et à Sophie de m'attendre et je suis allé le replacer dans son phare. Une fois dans la tourelle, je l'ai déposé dans sa caisse et je lui ai demandé pardon; je sais, c'est idiot de parler à un vieux cerfvolant, mais c'est comme ça. (Lévy, Le voleur d'ombres, 2010) 
Les occurrences (42) et (43), où l'énonciation de je sais $s_{1}$ ne laisse pas son interlocuteur continuer, illustrent également cette propriété:

42) Il passa la main sur sa blessure, la ramena vers lui, sombre sur sombre. Il chercha le saule pleureur des yeux, vit des pompiers à l'air fourbu. Le bruit de l'eau grésillant sur les braises.

- Charles...

- Charles aussi, je sais, le coupa Gaëlle. (Aubert, Funérarium, 2002)

43) - Il faudra la faire transporter dans mon atelier, dit-il. "Atelier»: un mot neutre, pour évoquer un travail neutre.

- Quand ça? demanda Andrieu.

- Aujourd'hui serait le mieux. Le temps a de l'importance.

- Je sais, le coupa Andrieu. (Aubert, Funérarium, 2002)

Dans (43), le stéréotype convoqué est explicite «le temps a de l'importance», mais dans (42) on fait appel, comme c'est souvent le cas, surtout lorsque je sai $s_{1}$ constitue à lui seul une énonciation, à un savoir partagé de forme «dire ce qui fait mal, ça fait mal», il vaut mieux ne pas dire ce qui est désagréable.

La stratégie propre à je sais ${ }_{1}$ consiste donc à établir la relation entre au moins quatre $p d v: p d v_{1}$, dont la source est un ex-locuteur (qui peut s'identifier au locuteur $\lambda$ ) et l'objet construit $\{p\} ; p d v_{2}$ dont la source est L et qui se présente comme prenant en charge $p d v_{1} ; p d v_{3}$ dont la source est un $O N$-locuteur et l'objet construit $\{q\} ; p d v_{4}$ dont la source est L et consiste dans la prise en charge de $p d v_{3}$ pour s'opposer à l'énonciation de $\{p\}$.

\section{Conclusion}

L'analyse montre qu'une optique sémantico-pragmatique, qui considère que la langue n'a pas pour fonction essentielle de transmettre des informations mais d'établir des rapports intersubjectifs, s'avère particulièrement efficace à expliquer la signification des marqueurs discursifs. En particulier, dans le cas de je sais et $_{1}$ tu sais $/$ tu sais $_{2}$, l'analyse a montré qu'il ne s'agit à aucun moment de simples marqueurs de structuration du discours mais qu'ils jouent toujours un rôle discursif dans l'agencement profond du discours, participant à la construction de stratégies discursives visant l'adhésion à des $p d v$, 
qui structurent les relations entre $\mathrm{L}$ et son allocutaire ainsi que la communauté linguistique (ON-locuteur) à laquelle il dit appartenir ou non.

\section{Bibliographie}

ANDERSEN H.-L. (1996), Verbes parenthétiques comme marqueurs discursifs, in Muller C. (éd.), Dépendance et intégration syntaxique. Subordination, coordination, connexion, Tübingen, Max Niemeyer Verlag, 307-315.

ANDERSEN H.-L. (2007), Marqueurs discursifs propositionnels, Langue Française 154, 13-28.

ANSCOMBRE J.-C. (1981), Marqueurs et hypermarqueurs de dérivation illocutoire: notions et problèmes, Cahiers de Linguistique Française 3, 75-124.

ANSCOMBRE J.-C. (2001), Le rôle du lexique dans la théorie des stéréotypes, Langages 142, 57-76.

ANSCOMBRE J.-C. (2013), Polyphonie et représentation sémantiques: notions de base, in Anscombre J.-C., Donaire M. L. \& Haillet P.P. (éds), Opérateurs discursifs du français. Éléments de description sémantique et pragmatique, Berne, Peter Lang, 11-32.

BLANCHE-BENVENISTE C. \& WILLEMS D. (2007), Un nouveau regard sur les verbes "faibles», Bulletin de la Société de Linguistique de Paris CII : 1, 217-254.

DAVOINE J.-P. (1981), Tu sais! c'est pas facile, Linguistique et Sémiologie, 10: L'Argumentation, Lyon, Presses Universitaires de Lyon, 109-124.

DONAIRE M.L. (à paraître), Je dirais, je ne saurais dire, comment dirais-je: s'agit-il vraiment d'un dire au conditionnel?, in Anscombre J.-C. \& Rouanne L. (éds), Histoires de dire. Petit glossaire des marqueurs formés sur le verbe dire, Berne, Peter Lang.

DOSTIE G. (2004), Pragmaticalisation et marqueurs discursifs. Analyse sémantique et traitement lexicographique, Bruxelles, De BoeckDuculot.

DOSTIE G. \& DE SÈVE S. (1999), Du savoir à la collaboration. Étude pragma-sémantique et traitement lexicographique de t'sais, Revue de Sémantique et Pragmatique 5, 11-35.

RIEGEL M. et al. (1994), Grammaire méthodique du français, Paris, Presses universitaires de France. 
SETTERKORN W. (1977), Pragmatique et rhétorique discursive, Journal of Pragmatics 1, 195-209.

TRAVERSO V. (1996), La conversation familière. Analyse pragmatique des interactions, Lyon, Presses universitaires de Lyon.

VINCENT D. (1993), Les ponctuants de la langue et autres mots du discours, Québec, Nuit Blanche Éditeur.

WILLEMS D. \& BLANCHE-BENVENISTE C. (2010), Verbes « faibles» et verbes à valeur épistémique en français parlé: il me semble, il paraît, j'ai l'impression, on dirait, je dirais, in Iliescu M., SillerRunggaldier H. \& Danler, P. (éds), Actes du XXV $V^{e}$ Congrès International de Linguistique et de Philologie Romane (Innsbruck 2007), Berlin/New York, De Gruyter, tome IV, 565-576.

Clapi: http://corpus.univ-lyon2.fr

Corpus de la parole: http://corpusdelaparole.huma-num.fr

Elicop: http://bach.arts.kuleuven.be/elicop

Eslo: http://eslo.huma-num.fr 